\title{
Особенности формирования замкнутых модовых структур в прямоугольных резонаторах на основе гетероструктур InGaAs/AIGaAs/GaAs для мощных полупроводниковых лазеров
}

\author{
(C) А.А. Подоскин, Д.Н. Романович, И.С. Шашкин, П.С. Гаврина, \\ 3.Н. Соколова, С.О. Слипченко, Н.А. Пихтин \\ Физико-технический институт им. А.Ф. Иоффе Российской академии наук, \\ 194021 Санкт-Петербург, Россия \\ E-mail: podoskin@mail.ioffe.ru
}

Поступила в Редакцию 29 декабря 2018 г.

В окончательной редакции 14 января 2019 г.

Принята к публикации 14 января 2019 г.

Работа посвящена исследованию особенностей формирования высокодобротных замкнутых мод, работающих на эффекте полного внутреннего отражения, в прямоугольных резонаторах большого размера (сотнитысячи длин волн) на основе лазерных гетероструктур InGaAs/GaAs/AlGaAs. В работе экспериментально исследованы особенности спектрального состава излучения и пространственных конфигураций модовых структур. Также рассмотрено влияние изменения накачки и температуры на модовый состав излучения.

DOI: $10.21883 /$ FTP.2019.06.47739.9058a

\section{1. Введение}

В настоящее время существует множество устройств фотоники, предназначенных для управления потоками оптического излучения с помощью резонаторных устройств различной конфигурации. Так, в системах обработки и передачи информации используются микрорезонаторы различных форм: дисковые, кольцевые, прямоугольные, многоугольные и их комбинации. В таких резонаторах формируется большое количество различных модовых структур с различной добротностью [1-6], позволяющих получать на выходе из устройства оптический поток с широким диапазоном заданных свойств. Типичные линейные размеры таких приборов составляют от сотен нанометров до десятка микрометров, что соответствует единицам-десяткам длин волн рабочего излучения. Прямоугольный резонатор как представитель наиболее простых для анализа геометрических форм даже при таких размерах характеризуется большим разнообразием модового состава $[1,2,6]$. Возможности управления характеристиками модовых структур резонаторов малого размера ограничены. С увеличением размеров разнообразие модового состава возрастает, однако при этом увеличиваются возможности по управлению этим составом. Исследования мод различной добротности в больших прямоугольных резонаторах с характерными размерами в тысячи длин волн (сотни мкм) на основе гетероструктур мощных полупроводниковых лазеров проводились в работах [7-9]. Была показана возможность переключения между низкодобротной модой Фабри-Перо (ФПМ) и высокодобротной замкнутой модой (3М) при управляемом изменении соотношения их внутренних оптических потерь. На основе управляемой конкуренции ФПМ и ЗМ была представлена оптическая ячейка, работающая как в режиме модулятора мощности ФПМ, так и в режиме ячейки памяти с необратимым бистабильным переключением между модовыми структурами. При этом исследований принципов формирования модовых конфигураций и управляемой пространственной селекции мод не проводилось. Управление модовым составом большого прямоугольного резонатора открывает новые возможности для создания источников лазерного излучения оптических систем передачи и обработки информации. В рамках данной работы проводится экспериментальное выявление спектральных и пространственных особенностей $3 \mathrm{M} \mathrm{в}$ больших прямоугольных резонаторах.

\section{2. Экспериментальные образцы}

Экспериментальные образцы изготавливались на основе гетероструктур InGaAs/GaAs/AlGaAs, типичных для мощных полупроводниковых лазеров полосковой конструкции на длину волны 900-1100 нм [10,11]. Конструкция гетероструктуры включала в себя эмиттеры $\mathrm{Al}_{0.4} \mathrm{Ga}_{0.6} \mathrm{As}$ толщиной 1.5 мкм, волновод $\mathrm{Al}_{0.3} \mathrm{Ga}_{0.7} \mathrm{As}$ толщиной 1.6 мкм, квантовую яму (КЯ) InGaAs толщиной 9 нм с длиной волны излучения 1040-1060 нм в спейсерах GaAs по 6 нм. КЯ расположена на расстоянии 0.6 мкм от р-эмиттера. Предварительная характеризация лазерной гетероструктуры с помощью образцов полупроводниковых лазеров с полосковым контактом 100 мкм показала внутренние оптические потери $1.6 \mathrm{~cm}^{-1}$, внутренний квантовый выход 97\% и характеристическую пороговую плотность тока $82 \mathrm{~A} / \mathrm{cm}^{2}$. В ходе постростовых операций по изготовлению экспериментальных образцов на пластине гетероструктуры формировались контактные полоски $\mathrm{AuZn} / \mathrm{Cr} / \mathrm{Au}$ шириной 200 мкм, образующие области активной токовой накачки. Пассивные части кристалла шириной 600 мкм 
оставляли свободными от металлического контакта, дополнительно производили стравливание контактного слоя. Со стороны подложки наносился сплошной металлический контакт $\mathrm{AuGe} / \mathrm{Cr} / \mathrm{Au}$. Из пластины путем скалывания по естественным плоскостям спайности формировались кристаллы (лазерные чипы) с размерами от $200 \times 200$ до $700 \times 700$ мкм и различным расположением активного полоска (как по центру с изначальной шириной в 200 мкм, так и с краев кристалла при ширине контактной области < 200 мкм). Лазерные кристаллы паяли с помощью припоя ПОС-61 на медные теплоотводы, совместимые с экспериментальной измерительной установкой.

\section{3. Эксперимент}

Схема экспериментальной установки изображена на рис. 1. Экспериментальная установка включала в себя медную термостабилизированную монтажную площадку, на которую с помощью термопасты устанавливался медный носитель с экспериментальным образцом, стабилизированный источник непрерывной накачки Agilent E3634, систему регистрации спектра излучения на основе спектроанализатора Thorlabs OSA-202, оптическую схему сбора излучения с грани кристалла и ввода в приемное волокно спектроанализатора, а также систему визуализации пространственной конфигурации ЗМ на основе инфракрасной (ИК) фотокамеры и оптической схемы для построения увеличенного изображения экспериментального образца. Для ввода излучения в приемное волокно спектроанализатора с помощью согласованной пары асферических линз строилось увеличенное изображение грани образца на торце волокна - таким образом обеспечивалась возможность селективного измерения спектра генерации от различных участков на грани экс-

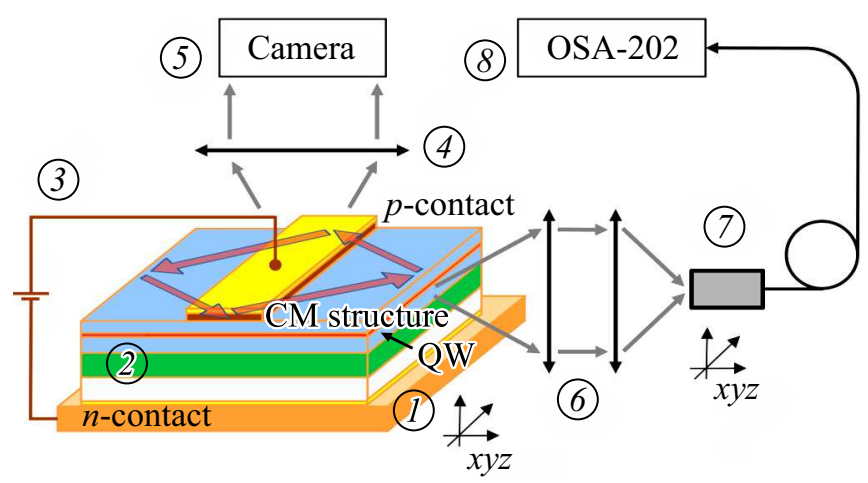

Рис. 1. Схема экспериментальной установки для анализа спектральных и пространственных характеристик замкнутых модовых структур (СМ). 1 - термостабилизированная площадка на трехкоординатном трансляторе, 2 - образец кристалла с КЯ $(\mathrm{QW}), 3$ - источник накачки, 4 - объектив, 5 - регистрирующая ИК камера, 6 - собирающий дублет асферических линз, 7 - вход приемного волокна спектроанализатора, 8 - спектроанализатор. периментального образца. Линейное увеличение схемы составляло 2.5, диаметр ядра волокна - 50 мкм.

Измерения спектров генерации 3М проводились для серии образцов с размерами сторон от 250 до 700 мкм (рис. 2). С увеличением размера кристалла и соответственно непрокачиваемой части кристалла пороговый спектр генерации смещался в сторону бо́льших длин волн и достигал 1055-1058 нм. Генерация ФПМ (при ее наличии) смещена относительно ЗМ в коротковолновую сторону на 15-20 нм [12]. На пороге генерации спектр $3 \mathrm{M}$ представлял собой одиночные лазерные линии. При этом в кристалле наблюдается возникновение характерной траектории 3М (рис. 2,a). Применение интерференционного фильтра с шириной полосы пропускания 10 нм и центральной длиной волны, соответствующей линиям 3М, приводит к значительному ослаблению яркости изображения с регистрирующей камеры. Это означает, что визуализация ЗМ происходит за счет спонтанной люминесценции от оптически накачанной КЯ лазерной гетероструктуры в области распространения пучка излучения 3М в свободных от контактов частях кристалла. С ростом тока накачки количество линий в спектре 3М возрастало, среди них можно было выделить серии эквидистантных линий в виде гребенок. Отдельные гребенки линий могут как располагаться на различных участках спектра, так и перекрываться (рис. 2, b). Наблюдаемые продольные межмодовые расстояния в гребенках можно описать соотношением

$$
\Delta \lambda=K \lambda^{2} /\left(n_{\mathrm{eff}} L_{r}\right),
$$

где $K$ - целое число, $\lambda$ - длина волны генерации в воздухе, $n_{\mathrm{eff}}$ - эффективный показатель преломления для фундаментальной (нулевой) моды лазерной гетероструктуры, $L_{r}$ - характерная длина участка траектории 3М в кристалле. В ходе исследований спектров установлено, что в качестве характерной длины $L_{r}$ может выступать отрезок траектории 3М между двумя отражениями от граней, сумма нескольких отрезков или общая длина всей замкнутой траектории. В простейшем случае с однократным отражением от каждой грани прямоугольного кристалла размера $a \times b$ характеристическая длина $L_{r}=\sqrt{a^{2}+b^{2}}$. В этом случае при $K=1$ межмодовое расстояние в ЗМ (1) соответствует межмодовому расстоянию резонатора Фабри-Перо, где $L_{r}$ - длина резонатора.

В одном образце наблюдались различные варианты визуальных конфигураций 3М. Число переотражений в замкнутой траектории может быть как минимальным, т.е. 4, так и существенно бо́льшим (до 10 и более). Визуальная ширина отдельной области свечения - от 5 до 30 мкм. В ходе исследований серии образцов установлено, что с увеличением размеров кристалла возрастает разнообразие 3М как по количеству спектральных компонент, так и по визуальным пространственным конфигурациям в виде множества отдельных закольцованных траекторий. 



Рис. 2. Замкнутая мода в кристалле $620 \times 700$ мкм с полосковым контактом шириной 200 мкм при накачке током 1.6 А: $a-$ фото пространственной конфигурации замкнутой моды в диапазоне длин волн $1000-1100$ нм; $b$ - типичный спектр генерации 3М, где в области длин волн 1056-1057 и 1058 наблюдаются отдельные гребенки с межмодовыми расстояниями 350 и 175 пм соответственно, а в области 1055.5-1056 нм - типичная картина взаимного наложения спектральных гребенок.

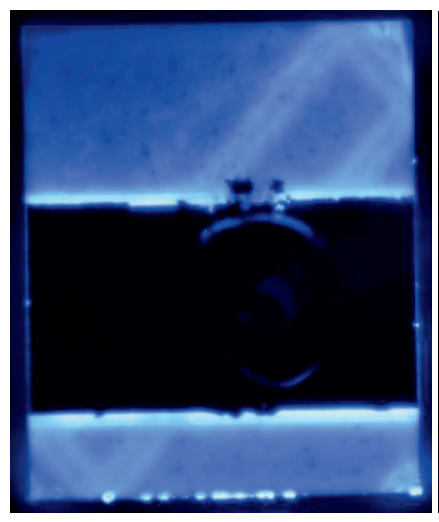

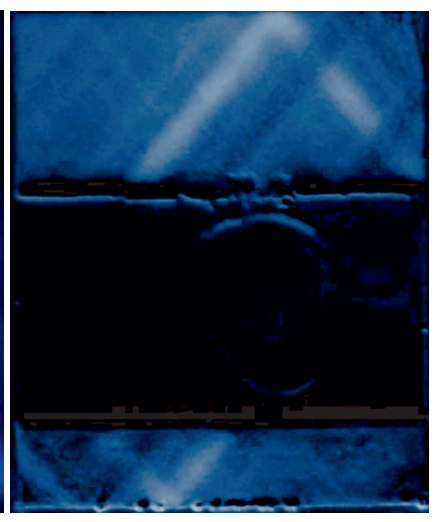

$b$

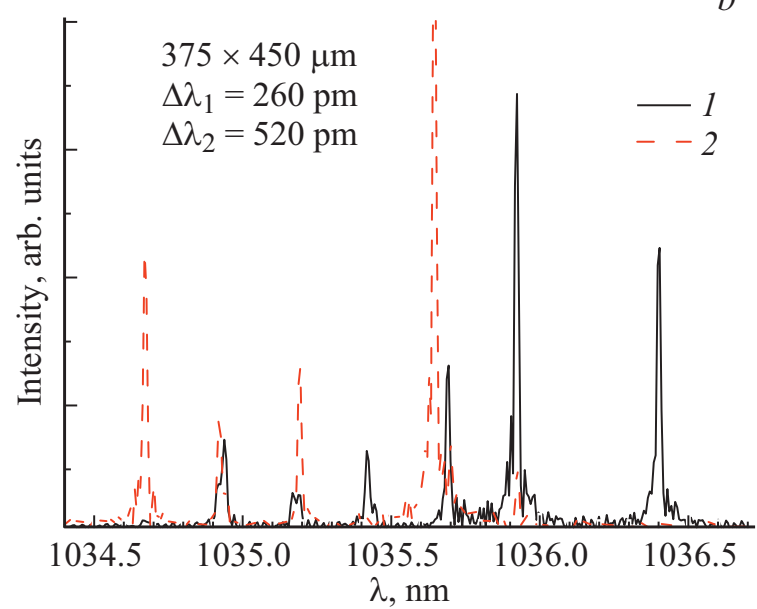

Рис. 3. Возникновение отдельной конфигурации замкнутой моды в кристалле $375 \times 400$ мкм с полосковым контактом шириной 200 мкм при изменении тока накачки: $a-$ фото с изображением пространственной конфигурации замкнутой моды в диапазоне длин волн 1000-1100 нм при токе накачки 212 (слева) и 213 мА (справа) (изображение получено при вычитании предыдущего изображения в качестве фона); $b$ - спектры генерации замкнутой моды при токах накачки 212 (1), 213 мА (2). Наблюдаемые межмодовые расстояния $\Delta \lambda_{1}=260$ и $\Delta \lambda_{2}=520$ пм.

На втором этапе эксперимента проводился анализ распределения вывода излучения из экспериментального образца. Для этого осуществлялось сканирование входным волокном спектроанализатора вдоль увеличенного изображения грани экспериментального образца. Области максимальной интенсивности выходящего излучения расположены в углах кристалла. Также наблюдались локальные максимумы в области дефектов скола на грани кристалла.

Для дальнейшего анализа параметров конфигураций 3М были проведены измерения спектров генерации с визуализацией пространственной структуры 3М при различных токах накачки и различных температурах. В ходе исследований установлено, что как изменение тока накачки, так и изменение температуры экспериментального образца оказывают значительное влияние на разнообразие структур 3М. На рис. 3 показано, что при возникновении с ростом тока накачки новой закольцованной конфигурации ЗМ в экспериментальном кристалле появляется новая дополнительная компонента (отдельная линия или гребенка линий) в спектре лазерной генерации. 


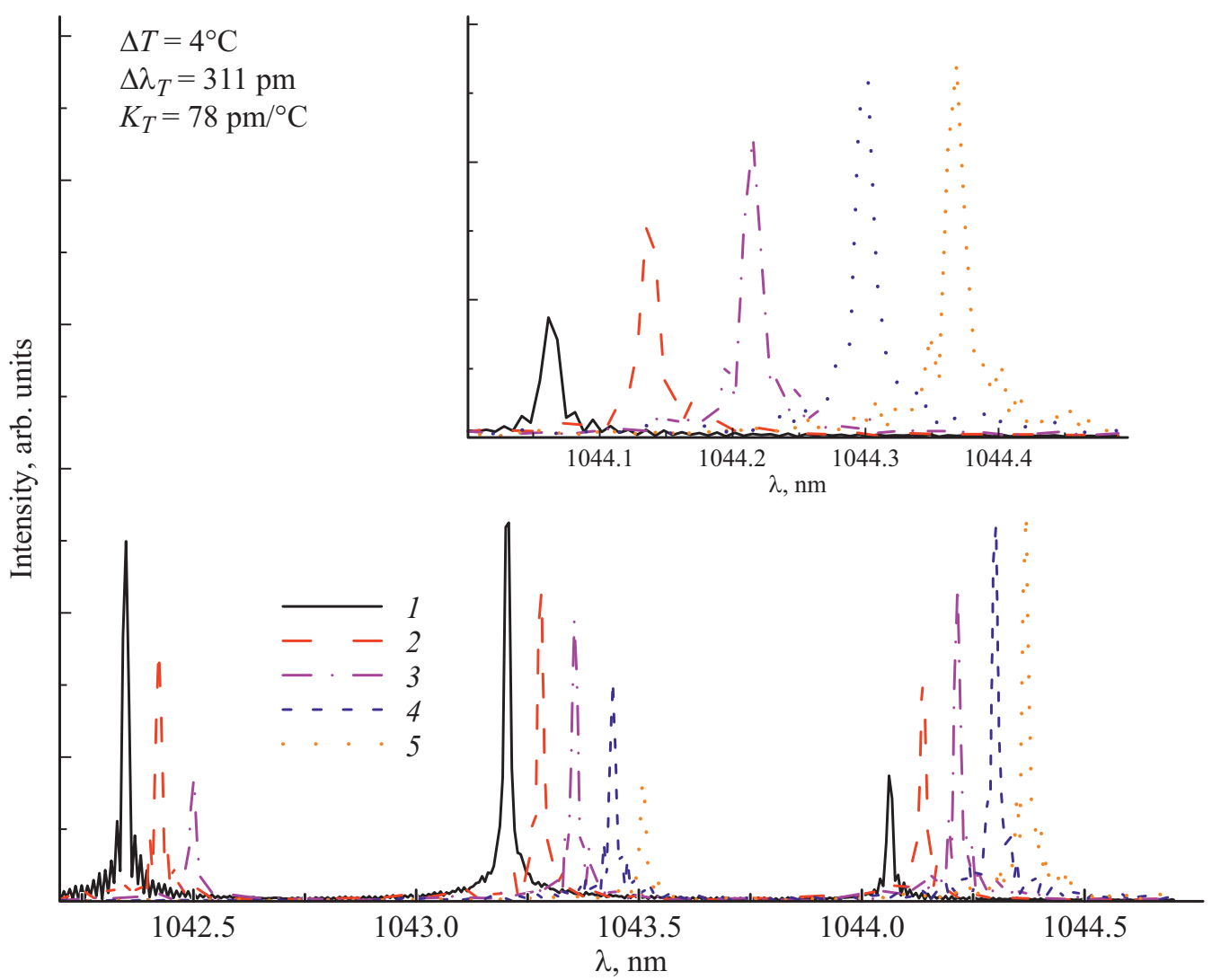

Рис. 4. Спектры генерации замкнутой моды в кристалле $460 \times 580$ мкм с полоском шириной 200 мкм при токе накачки 870 мА и температурах теплоотводов ${ }^{\circ} \mathrm{C}: 1-22,2-23,3-24,4-25,5-26$. На вставке - увеличенное изображение температурной динамики отдельной линии спектра.

Дополнительно получена оценка температурной стабильности спектров генерации ЗМ. С ростом температуры на $\Delta T$ при фиксированном токе накачки происходит деформация спектра 3М. Деформацию спектра можно представить в виде суммы двух составляющих. Первая составляющая - общее смещение спектра в длинноволновую область в связи с изменениями в спектре усиления КЯ и температурными изменениями пороговых условий лазерной моды [13]. Вторая составляющая сдвиг отдельных линий в длинноволновую область, обусловленный температурным расширением кристалла и увеличением показателя преломления волноводного слоя гетероструктуры. Вторую составляющую можно описать как $\Delta \lambda_{T}=\lambda_{0} \Delta T\left(K_{T}+K_{n}\right)$, где $\lambda_{0}-$ исходная длина волны, $K_{T}$ и $K_{n}-$ коэффициент линейного расширения кристалла и коэффициент линейного увеличения показателя преломления с температурой. Для волновода $\mathrm{Al}_{0.3} \mathrm{Ga}_{0.7} \mathrm{As}$ и подложки GaAs получено смещение линий на $\sim(78 \pm 5)$ пм/К (рис. 4$)$.

\section{4. Заключение}

Проведено экспериментальное исследование особенностей формирования 3М структур в прямоугольных резонаторах большого размера на основе квантово- размерных лазерных гетероструктур. Показано, что спектр генерации 3М состоит из множества компонент в виде серий эквидистантных линий - гребенок (в том числе с вырождением в одиночные линии). Соотношение для межмодового расстояния в гребенке аналогично случаю ФПМ, однако в качестве характеристической длины может выступать как общая длина траектории $3 \mathrm{M}$, так и длина отдельных ее участков или их линейные комбинации. С увеличением размера кристалла возрастает разнообразие как пространственных конфигураций 3М, так и спектральных компонент. Каждая отдельная конфигурация 3М, возникающая в кристалле, соответствует серии линий (гребенке) в спектре. В кристаллах большого размера наблюдается одновременная генерация различных гребенок, как спектрально раздельных, так и перекрывающихся.

Работа выполнена при поддержке программы РФФИ „а“ грант 18-02-00835 А.

\section{Список литературы}

[1] Wei-Hua Guo, Yong-Zhen Huang, Qiao-Yin Lu, Li-Juan Yu. IEEE J. Quant. Electron., 39 (12), 1563 (2003).

[2] M.Y. Tang, S.S. Sui, Y.D. Yang, J.L. Xiao, Y. Du, Y.Z. Huang. Opt. Express, 23 (21), 27739 (2015). 
[3] H.T. Hattori. JOSA B, 25 (11), 1873 (2008).

[4] Y.D. Yang, Y.Z. Huang. IEEE J. Quant. Electron., 43 (6), 497 (2007).

[5] Y.D. Yang, Y.Z. Huang. J. Phys. D: Appl. Phys., 49 (25), 253001 (2016).

[6] A.W. Poon, F. Courvoisier, R.K. Chang. Optics Lett., 26 (9), 632 (2001).

[7] S.O. Slipchenko, A.A. Podoskin, N.A. Pikhtin, I.S. Tarasov. Laser Phys., 24 (10), 105001 (2014).

[8] А.А. Подоскин, И.С. Шашкин, С.О. Слипченко, Н.А. Пихтин, И.С. Тарасов. ЖТФ, 43 (2), 31 (2017).

[9] А.А. Подоскин, И.С. Шашкин, С.О. Слипченко, Н.А. Пихтин, И.С. Тарасов. ФТП, 49 (8), 1108 (2015).

[10] N.A. Pikhtin, S.O. Slipchenko, Z.N. Sokolova, A.L. Stankevich, D.A. Vinokurov, I.S. Tarasov, Z.I. Alferov. Electron. Lett., 40 (22), 1413 (2004).

[11] P. Crump, G. Erbert, H. Wenzel, C. Frevert, C.M. Schultz, K.-H. Hasler, R. Staske, B. Sumpf, A. Maaßdorf, F. Bugge, S. Knigge, G. Trankle. IEEE J. Select. Top. Quant. Electron., 19 (4), 1501211 (2013).

[12] S.O. Slipchenko, A.A. Podoskin, N.A. Pikhtin, Z.N. Sokolova, A.Y. Leshko, I.S. Tarasov. Semiconductors, 45 (5), 663 (2011).

[13] L.A. Coldren, S.W. Corzine. Diode lasers and photonic integrated circuits (N.Y., John Wiley \& Sons Inc., 1995).

Редактор Л.В. Шаронова

\title{
Closed mode formation features in rectangular resonators based on GaAs/AIGaAs/GaAs heterostructures for powerful semiconductor lasers
}

A.A. Podoskin, D.N. Romanovich, I.S. Shashkin, P.S. Gavrina, Z.N. Sokolova, S.O. Slipchenko, N.A. Pikhtin

loffe Institute, 194021 St. Petersburg, Russia

\begin{abstract}
The work is devoted to the study features of formation working on the total internal reflection effect high-quality of closed modes in rectangular resonators of large size (up to thousands wavelengths) based on $\mathrm{InGaAs} / \mathrm{GaAs} / \mathrm{AlGaAs}$ laser heterostructures. Paculiarities of the spectral composition and the spatial configurations of closed mode structures are experimentally investigated. The presence of frequency combs in the spectra is demonstrated and their correspondence to separate spatial configurations of closed modes is shown. The effect of a change in pumping and temperature on the mode composition is also considered.
\end{abstract}

